\title{
Flow-cytometry phenotypic assessment of immune cell subsets reflecting function for the identification of breast cancer patients receiving vaccine plus docetaxel with longer progression-free survival
}

\author{
Italia Grenga*, Renee N Donahue, James L Gulley, Christopher Heery, Ravi A Madan, Jeffrey Schlom,
} Benedetto Farsaci

From Society for Immunotherapy of Cancer 28th Annual Meeting

National Harbor, MD, USA. 8-10 November 2013

\section{Purpose}

Aim of this study was to assess whether specified immune cells subsets at baseline could help identifying patients with longer progression-free survival (PFS) in a clinical trial of metastatic breast cancer patients receiving docetaxel \pm vaccine.

\section{Methods}

We applied flow-cytometer analysis of PBMCs harvested before treatment from patients $(n=43)$ enrolled in a small randomized phase II study of docetaxel alone $(n=20)$ or in combination with PANVAC ${ }^{\mathrm{TM}}-\mathrm{V}$ (Vaccinia) and PAN$\mathrm{VAC}^{\mathrm{TM}}$-F (Fowlpox) encoding for the tumor-associated antigens CEA and MUC-1, along with a TRIad of COstimulatory Molecules (B7-1, ICAM-1, and LFA-3; called TRICOM) $(\mathrm{n}=23)$. As criterion 1 , we analyzed the frequency standard immune subsets, i.e. CD4, CD8, NK, Treg, MDSC, and their ratios. As criterion 2, we measured phenotypes indicating immune function, i.e. central memory $\mathrm{T}$ lymphocytes, $\mathrm{T}$ cells expressing at $\geq 2$ suppressive markers among CTLA-4, PD1, TIM3, and 2B4, CD49d $\mathrm{d}^{-}$Tregs, lin ${ }^{-}$MDSCs, CD56 ${ }^{\mathrm{br}} \mathrm{CD} 16^{-} \mathrm{NK}$ cells, and their ratios. An immunoscore was generated based on the analysis of tertiles. Log-Rank Kaplan Meier analysis was applied to evaluate differences of PFS between patients with low- and- high immunoscore.

\section{Results}

The predetermined immunoscore based on phenotypes indicating immune function allowed discrimination between patients with longer PFS vs. shorter PFS in vaccine plus docetaxel arm $(\mathrm{p}<0.001, \mathrm{HR}=0.049)$ but not in docetaxel alone $\operatorname{arm}(\mathrm{p}=0.875 ; \mathrm{HR}=0.926)$.

\section{Conclusions}

The calculation of an immunoscore based on a flow-cytometer screening of predetermined immune subsets indicating immune function from PBMCs before treatment could be a potential useful tool for the identification of patients that can benefit from combination immunotherapy.

Published: 7 November 2013

doi:10.1186/2051-1426-1-S1-P51

Cite this article as: Grenga et al:: Flow-cytometry phenotypic assessment of immune cell subsets reflecting function for the identification of breast cancer patients receiving vaccine plus docetaxel with longer progressionfree survival. Journal for ImmunoTherapy of Cancer 2013 1(Suppl 1):P51. 